\title{
Características clínicas, factores de riesgo y perfil de susceptibilidad de las infecciones por micobacterias documentadas por cultivo, en un hospital universitario de alta complejidad en Medellín (Colombia)
}

\section{Franco E. Montufar Andrade, Carolina Aguilar Londoño, Carolina Saldarriaga Acevedo, Alicia Quiroga Echeverri, Carlos E. Builes Montaño, Miguel A. Mesa Navas, Olga L. Molina Upegüi, y John J. Zuleta Tobón}

Clinical features, risk factors and susceptibility profile of mycobacterial infections documented by culture in a university hospital of high complexity in Medellin (Colombia)

Introduction: Tuberculosis (TB) remains an entity of high prevalence and mortality worldwide. The rising drug resistance is a public health problem. Besides, non-tuberculosis mycobacterial (NTM) infections are described with increasing frequency in areas of high prevalence of TB. Objectives: To determine epidemiological, clinical and microbiological characteristics of mycobacterial infections documented by culture. Materials and Methods: An observational, descriptive study in hospitalized patients. Results: M. tuberculosis complex was identified in $90,9 \%$ of 187 patients; $9,1 \%$ had NTM, $64 \%$ were male and the mean age was 40 years (range $1-88$ years). The main co-morbidities were HIV / AIDS (23.5\%), use of corticosteroids (13.3\%) and chronic kidney disease (9.6\%). Clinical forms were pulmonary (56.6\%), extra-pulmonary (23.9\%) and disseminated (19.2 The most common extra-pulmonary compromise was nodal (7.4\%) and gastrointestinal (7\%). 10.6\% of M. tuberculosis were multidrugresistant (MDR) and 2.12\% had extended drug resistance (XDR). Mycobacterium avium and M. abscessus were the most frequent NTM. Overall mortality was $10 \%$. Conclusions: In our study immune suppression is the main risk factor for extrapulmonary and disseminated disease. Resistance, MDR and XDR is higher in inpatients with TB. MNT infections are not uncommon in our country.

Key words: Tuberculosis; Mycobacterium; infections; nontuberculous mycobacteria

Palabras clave: Tuberculosis; micobacterias; infección; micobacterias no tuberculosas.

\section{Introducción}

$\mathrm{T}$ uberculosis (TBC), considerada un problema de salud pública mundial, es la segunda causa de muerte por enfermedades infecciosas después de la infección por el virus de inmunodeficiencia humana (VIH). La Organización Mundial de la Salud (OMS) estimó que 8,6 millones de personas desarrollaron TBC y 1,3 millones murieron durante el año 2012. En estas muertes se incluyen 320.000 personas con infección por VIH. La incidencia de TBC en el mundo es variable, está alrededor de 1.000 casos por 100.000 habitantes en Sud-África y tan sólo de 10 por 100.000 en algunas regiones de las Américas, Europa, Japón, Australia y Nueva Zelanda. La mayoría de los casos en 2012 se presentaron en el Suroeste Asiático (29\%), África (27\%) y regiones del pacífico (19\%); de hecho, India y China aportaron el 26 y $12 \%$ del total de casos, respectivamente. La tasa de mortalidad se ha reducido en $45 \%$ desde 1990 y la meta es disminuirla en 50\% para el 2015. De igual forma, la tasa de incidencia de TBC descendió en 37\% desde 1990 hasta la fecha y se proyecta que esta reducción sea de $50 \%$ en el 2015. Se estima que 1,1 millón de personas (13\%) de los 8,6 millones que desarrollaron TBC en el 2012 eran portadores de VIH, 450.000 desarrollaron TBC multidrogo-resistencia (TBC-MDR) y cerca de 170.000 murieron por ella ${ }^{1}$.

En Colombia se reportan cerca de 12.000 casos nuevos por año, con una incidencia entre 24 y 26 casos por 100.000 habitantes, $16,5 / 100.000$ para TBC con baciloscopia positiva y $4,6 / 100.000$ para TBC extrapulmonar. Cada año se reportan alrededor de 1000 muertes por TBC, a pesar de que la tasa de mortalidad ha disminuido cerca de $40 \%$, pasando de 3,45 casos por 100.000 en 1999 a 2,1 casos en 2010. La tasa de co-infección por VIH en el país está entre 10 y $15 \%$, con una cobertura de la prueba de infección por VIH de $61 \%$ en los casos de TBC. En Colombia, cerca de $10 \%$ de los casos nuevos de TBC se
Hospital Pablo Tobón Uribe Medellín, Colombia. Departamento de Medicina Interna (FEMA). Departamento de Bacteriología (OLMU). Unidad de Investigación (JJZT) Universidad Pontificia Bolivariana.

Residentes de Medicina Interna (CAL, CSA, AQE, CEBM, MAMN).

Los autores declaran que no tienen conflictos de intereses ni recibieron dinero de alguna institución para esta publicación. El Hospital Pablo Tobón Uribe ofreció al grupo investigador todo el apoyo logístico necesario para llevar a cabo la recolección de datos, análisis estadístico y elaboración del artículo.

Recibido: 28 de noviembre de 2013 Aceptado: 31 de octubre de 2014

Correspondencia a: Franco E. Montufar Andrade. frmontufar@yahoo.com. 
presentaron bajo los 15 años de edad y, en uno de sus departamentos, Antioquia, para el 2011 se notificaron 2.553 casos nuevos de TBC pulmonar y extrapulmonar, con una incidencia de 46,6/100.000, con tendencia ascendente desde $2009^{2}$. En el sistema de seguridad social de Colombia el tratamiento supervisado o directamente observado (DOTS) está implementado por resolución del Ministerio de la Protección Social desde el año 2000 y se estima una cobertura aproximada de $60 \%$. Desde este mismo año, se implementa además la vigilancia activa de la co-infección TBC-VIH, siendo obligatorio y mandatorio que todo paciente con TBC sea evaluado para VIH y que a todo paciente con VIH se haga búsqueda activa de TBC. Estas decisiones quedan refrendadas en el plan estratégico Colombia Libre de Tuberculosis 2010-2015 $5^{3,4}$.

El riesgo de desarrollar TBC es mucho mayor en adictos o usuarios de drogas intravenosas, personas quienes trabajen o vivan en hacinamiento (cárceles, hogares de cuidado crónico), los provenientes de zonas de alta incidencia, la co-infección con VIH y otros estados de inmunosupresión tales como trasplante de órganos, diabéticos y enfermedad renal crónica ${ }^{5,6}$. Se ha considerado también por algunos autores el tabaquismo como factor de riesgo ${ }^{6,7}$. Actualmente, el incremento en el uso de medicamentos como los antagonistas del factor de necrosis tumoral (FNT) en enfermedades como la artritis reumatoide (AR), psoriasis y enfermedad de Crohn, han aumentado la incidencia de infección por $M$. tuberculosis, especialmente las formas extrapulmonares y diseminada ${ }^{8}$. En el grupo de pacientes con inmunosupresión de base y especialmente en pacientes con infección por VIH que tengan infección latente, el riesgo de desarrollar enfermedad, y en especial formas diseminadas o extrapulmonares, se incrementa varias veces, con un riesgo anual mayor que en el inmunocompetente 9 .

Según el proyecto global de vigilancia en resistencia a TBC de la OMS, para el 2012 a nivel mundial se documentó TBC-MDR en 3,6\% de los casos nuevos y 20\% en aquellos que habían recibido tratamiento. Adicionalmente, en los casos nuevos de TBC la resistencia a algún fármaco fue de $11 \%$ y se estima que en promedio, $9,6 \%$ de los casos de TBC-MDR tienen TBC-XDR. Cuando se compara la mortalidad durante el tratamiento ésta es mucho mayor y varía entre 56 y $64 \%$ en TB-XDR comparada con TB-MDR ${ }^{10,11,14}$. Esta tendencia en el incremento de la resistencia a fármacos ha hecho que en nuestro sistema de salud y seguridad social colombiano se recomiende realizar cultivos y pruebas de susceptibilidad a todas las muestras obtenidas de pacientes con infección VIH o en inmunosupresión de otra causa, en los contactos de pacientes con TBC-MDR o TBC-XDR* que enferman, en los pacientes con fallas en el tratamiento o recaídas $\mathrm{y}$ en todas las muestras obtenidas por broncoscopia ${ }^{1-4}$.

\footnotetext{
*Ver definiciones en Metodología.
}

Aunque la infección por $M$. tuberculosis es evidentemente la más importante desde el punto de vista epidemiológico, cada día se describen con más frecuencia las infecciones causadas por micobacterias no tuberculosas $(\mathrm{MNT})^{12}$. Los aislamientos del complejo Mycobacterium avium (CMA) son los más frecuentes seguidos de las micobacterias de crecimiento rápido y de M. kansasii ${ }^{12,13}$

Considerando entonces el panorama mundial de TBC, sus factores de riesgo y el incremento de resistencia a los fármacos anti-tuberculosos, pretendemos además de analizar las características socio-demográficas y clínicas, caracterizar el perfil de susceptibilidad de las infecciones por micobacterias en pacientes hospitalizados y aportar datos epidemiológicos a la región de las Américas.

\section{Metodología}

Estudio descriptivo que incluyó pacientes hospitalizados con infección por micobacterias, confirmadas por cultivo, en el período comprendido entre enero 2004 y diciembre 2011 en el Hospital Pablo Tobón Uribe, institución de enseñanza universitaria, centro de referencia de alta complejidad de la ciudad de Medellín (Antioquia).

Para el diagnostico de infecciones por micobacterias se realizan tinciones directas para bacilos ácido-alcohol resistentes como Ziehl-Neelsen, Ziehl-Neelsen modificado y tinción de auramina rodamina. Las siembras de las muestras para el aislamiento microbiológico durante los años 2004 a 2007 se realizaron en el medio de cultivo sólido Ogawa Kudoh (por duplicado cada muestra) y a partir del 2008 se realiza la siembra en un tubo de Ogawa Kudoh y otra en un tubo de MGIT; método automatizado BACTEC $^{\text {тм }}$ MGIT $^{\text {тм }}$, equipo de BectonDickinson. Para las pruebas de susceptibilidad y de identificación las muestras se remitieron a los laboratorios de referencia de nuestra ciudad; la Corporación de Investigaciones Biológicas (CIB) y/o al laboratorio Departamental de Salud Pública. En la CIB las pruebas de sensibilidad hasta el año 2009 se realizaban con el método de proporciones en agar, actualmente las realizan con MGIT. En el Laboratorio Departamental de Salud Pública las pruebas de susceptibilidad las realizan con el método de proporciones múltiples. Para la diferenciación de $M$. bovis y M. tuberculosis se realizaron pruebas bioquímicas de reducción de nitratos a nitritos, catalasa, pirazinamida y 2 trocarbofeno; luego se les remitió al Instituto Nacional de Salud para su confirmación. Actualmente, para diferenciación de especies se utilizan pruebas de biología molecular. Para identificar MNT, se utilizaron los medios de cultivo Lowestein Jensen, capa delgada y a partir del año 2005, MGIT. Para la identificación de especies se realizaron pruebas bioquímicas y pruebas moleculares. En los últimos dos años se realizan pruebas de identificación molecular para TBC-MDR, mediante la mutaciones del 
gen rpoB para rifampicina o la prueba de GenXpert.

Las definiciones de resistencia, multidrogoresistencia y resistencia extendida utilizadas en nuestro trabajo fueron las determinadas por la Organización Mundial de la salud (OMS). Tuberculosis Multidrogoresistente (TBC-MDR) se definió como la resistencia expresada por M. tuberculosis a isoniazida y rifampicina y TBC extremadamente resistente (TBC-XDR) si además de la resistencia expresada por $M$. tuberculosis a isoniazida y rifampicina, también se expresa resistencia quinolonas y al menos a uno de los tres medicamentos inyectables de segunda línea (capreomicina, kanamicina y amikacina) $)^{14}$

El listado de pacientes se obtuvo a partir del sistema de información de la institución que cuenta con profesionales que codifican hasta 20 diagnósticos y todos los procedimientos realizados a todos los pacientes hospitalizados. La información se obtuvo de las historias clínicas electrónicas de la institución y de los registros de microbiología que se mantienen en el programa Whonet.

Los datos se ingresaron en una hoja de cálculo de Excel y se analizaron con el programa SPSS 12. Las variables cuantitativas se presentan con media o mediana, según la distribución de los datos, y rango, las cualitativas con número absoluto y proporción. Se utilizó la prueba $\chi^{2} \mathrm{de}$ asociación para la diferencia de manifestaciones clínicas. Para implementar la investigación se contó con la aprobación del Comité de Investigación y Ética en Investigación del Hospital Pablo Tobón Uribe y, de acuerdo al artículo 11 de la Resolución 008430 de 1993 del Ministerio de Salud de Colombia, es una investigación sin riesgo para el paciente, motivo por el cual no requiere consentimiento informado.

\section{Resultados}

De 187 pacientes con cultivo positivo para micobacterias, 90,9\% tenían complejo $M$. tuberculosis y $9,1 \%$ MNT. Todos correspondían a pacientes colombianos de raza mestiza, con una edad promedio de 40 años (rango 1-88 años), $12,4 \%$ menores de 18 años y $64,7 \%$ hombres. El 53,4\% están inscritos en el régimen contributivo del Sistema de Seguridad Social en Salud del país, es decir pertenecen al grupo de población económicamente activa y cotizante al sistema de salud, el resto, $46,6 \%$, pertenecen a poblaciones de bajos recursos económicos en los cuales el estado subsidia la atención en salud.

Cuarenta y cuatro de 161 pacientes $(27,3 \%)$ de los que se cuenta con la información, tenían co-infección con VIH y las formas de presentación clínica eran diferentes entre ellos. La presentación extra-pulmonar y diseminada fue más frecuente en la población con infección por VIH $(42,6$ vs $13,5 \%$ p < 0,001) y la forma pulmonar en la población sin infección por $\operatorname{VIH}(65,9$ vs $21 \%$, p < 0,001).
Las formas extra-pulmonares más frecuentes fueron la ganglionar (18,2\%), gastrointestinal $(6,8 \%)$, ósea $(4,5 \%)$, meníngea $(4,5 \%)$ y cutánea $(2,3 \%)$.

\section{Infecciones por complejo Mycobacterium tuberculosis}

La edad promedio de este subgrupo de 170 pacientes fue de 39,7 años, (rango 1-88). El 65,3\% fueron hombres y $12,4 \%$ tenían menos de 18 años. El 98,8\% de las infecciones por complejo $M$. tuberculosis fueron causadas por M. tuberculosis y 1,2\% por M. bovis. Los factores de riesgo, las co-morbilidades y los medicamentos inmunosupresores usados por los pacientes se presentan en la Tabla 1. En la Tabla 2 están las formas de presentación clínica; el compromiso pulmonar es el más frecuente. Los dos casos de $M$. bovis se presentaron en dos niños: un varón y una mujer (1 y 11 años, respectivamente); uno tuvo enfermedad diseminada y el otro compromiso osteo-articular.

La mediana de la estancia hospitalaria de estos pacientes fue de 12 días (rango 0-193), 19,4\% requirió atención en UCI, con una mediana de estancia en ella de 9 días (rango 1-100). Se formuló e inició tratamiento anti-tuberculoso dentro de la institución en 137 (80,6\%) debido a que los otros pacientes no tenían la confirmación de TBC al momento del egreso y los estudios preliminares habían sido negativos. El esquema de tratamiento anti TBC más utilizado fue el recomendado en el sistema de seguridad social con medicamentos de primera línea: isoniazida, rifampicina, pirazinamida y etambutol en 110/137 (80,3\%). En los otros pacientes los esquemas se individualizaron acorde con los perfiles de susceptibilidad y la presencia de co-morbilidades. La mortalidad fue de $8,7 \%$ en 149 pacientes de quienes se conoció el desenlace.

Treinta y siete de los 146 pacientes de los que se cuenta con la información tenían co-infección con VIH, con un recuento promedio de LTCD4 de 115,4 céls/ml (rango 0-429) en los 26 en que se midió y una carga viral promedio de 324.382 copias/ml (rango $40-1.253 .817$ ) en los 22 que tienen este dato.

El perfil de susceptibilidad a los medicamentos antituberculosos se evaluó en 47 de 170 casos; 26 aislados de M. tuberculosis fueron sensibles a los fármacos de primera línea $(55,3 \%), 5(10,6 \%)$ fueron catalogados como TBCMDR y sólo se encontró un paciente $(2,12 \%)$ con TBCXDR. En los otros $17(36,17 \%)$ se encontró resistencia a alguno de los medicamentos de primera línea. En la Tabla 4 se presentan las susceptibilidades específicas.

De los 130 pacientes a quienes se les realizó radiografía de tórax al ingreso, 76 presentaban infiltrados pulmonares, clasificados como nodulares en $22(16,9 \%)$, alveolares en $18(13,8 \%)$, mixtos en $18(13,8 \%)$, reticulares en 14 $(10,8 \%)$ y miliares en $4(3,1 \%)$. De 66 pacientes a quienes 
Tabla 1. Características de la población según el tipo de infección tuberculosa. Hospital Pablo Tobón Uribe, enero 2004 - diciembre 2011

\begin{tabular}{|c|c|c|c|c|}
\hline \multirow[b]{2}{*}{ Características } & \multicolumn{2}{|c|}{ Complejo M. tuberculosis } & \multicolumn{2}{|c|}{ MNT } \\
\hline & $\mathrm{n}: 170$ & $\%$ rango & $\mathrm{n}: 17$ & $\%$ rango \\
\hline Edad (años) & $39,7 \pm 21$ & $1-88$ & $38,4 \pm 19,2$ & $4-75$ \\
\hline Sexo masculino & $112 / 187$ & 65,9 & 9 & 52,9 \\
\hline \multicolumn{5}{|l|}{ Factores de riesgo } \\
\hline Alcoholismo & $16 / 104$ & 15,4 & 2711 & 18,2 \\
\hline Tabaquismo & 23/105 & 21,9 & $1 / 11$ & 9,1 \\
\hline Contactos con sintomáticos respiratorios & 9/42 & 21,4 & 0 & 0 \\
\hline Procedimientos estéticos & & & 2 & 11,76 \\
\hline Drogas inhaladas & $5 / 69$ & 7,2 & 0 & 0 \\
\hline Usuarios de drogas intravenosas & $3 / 67$ & 4,5 & 0 & 0 \\
\hline \multicolumn{5}{|l|}{ Antecedentes } \\
\hline Infección por VIH & 37 & 21,76 & 7 & 41,17 \\
\hline Promedio de LCD4 (céls/mm³) & 115,42 & $1-439$ & 25 & $1-50$ \\
\hline Carga viral VIH (copias ARN/ml) & 162.057 & $40-1.253 .817$ & 83.344 & $69-100.000$ \\
\hline Uso crónico de corticosteroides & $23 / 146$ & 15,8 & $2 / 15$ & 13,3 \\
\hline Uso de inmunosupresores & $21 / 170$ & 12,4 & $3 / 17$ & 17,7 \\
\hline - Calcineurínicos & 4 & 23,52 & 1 & 5,88 \\
\hline - Calcineurínicos + micofenolato & 5 & 29,41 & 0 & 0 \\
\hline - Azatioprina + calcineurínico & 3 & 17,64 & 0 & 0 \\
\hline - Quimioterapia & 2 & 11,76 & 1 & 5,88 \\
\hline - Azatioprina & 1 & 0,58 & 1 & 5,88 \\
\hline - Micofenolato & 2 & 11,76 & 0 & 0 \\
\hline - Metrotrexato + anti FNT & 2 & 11,76 & 0 & 0 \\
\hline - Inhibidores de la tirosinkinasa & 1 & 0,58 & 0 & 0 \\
\hline - Azatioprina + rituximab & 1 & 0,58 & 0 & 0 \\
\hline Tuberculosis previa & $17 / 146$ & 11,6 & $3 / 15$ & 20,0 \\
\hline Enfermedad renal crónica & $15 / 146$ & 10,3 & $3 / 15$ & 20,0 \\
\hline Diabetes mellitus & $12 / 147$ & 8,2 & $4 / 15$ & 26,7 \\
\hline Trasplante de órgano sólido & $13 / 146$ & 8,9 & $2 / 15$ & 13,3 \\
\hline Neoplasias & $10 / 146$ & 6,8 & $3 / 15$ & 20,0 \\
\hline Estancia hospitalaria & 95,8 & $1-193$ & 146,4 & $1-193$ \\
\hline Requerimiento $\mathrm{UCl}$ & $34 / 141$ & 24,1 & $3 / 14$ & 21,4 \\
\hline Promedio de estancia en UCl & 3,3 & $1-100$ & 3,7 & $1-47$ \\
\hline \multicolumn{5}{|l|}{ Complicaciones } \\
\hline Falla respiratoria & $23 / 141$ & 16,2 & 1 & 7,1 \\
\hline Nefrotoxicidad & $11 / 141$ & 7,8 & 0 & 0 \\
\hline Hepatotoxicidad & $13 / 141$ & 9,2 & 0 & 0 \\
\hline FOM & $10 / 140$ & 7,1 & 0 & 0 \\
\hline Mortalidad & $13 / 149$ & 8,7 & 4 & 26,7 \\
\hline
\end{tabular}

se les realizó tomografía computarizada de tórax de alta resolución (TACAR), 22 (33,8\%) tenían compromiso multilobar, 27 (40,9\%) efusión pleural y $13(19,7 \%)$ lesiones cavitarias. Con respecto a los infiltrados en el TACAR, 28 $(42,4 \%)$ tuvieron infiltrado nodular, $13(19,7 \%)$ alveolar y $3(4,5 \%)$ infiltrados miliares (Figuras 1 y 2 ).

Se realizó TAC y/o RM de cráneo a 35 pacientes y fue normal en 50,0\%. En tres pacientes se identificaron lesiones compatibles con tuberculomas, dos de los cuales consultaron por síntomas neurológicos y uno tuvo alteración neurológica en el examen físico de ingreso dado por signos meníngeos. Tres pacientes presentaban hidrocefalia.

De 141 pacientes de los que se cuenta con la información, $23(16,3 \%)$ presentaron falla respiratoria, 13 $(9,2 \%)$ hepatotoxicidad medicamentosa, $11(7,8 \%)$ falla renal medicamentosa y $10(7,1 \%)$ falla orgánica múltiple.

\section{Infecciones por micobacterias no tuberculosas (MNT)}

La edad promedio de los 17 pacientes $(9,1 \%)$ con infección por MNT fue 38,4 años (rango 4-75 años). 
Tabla 2. Formas de presentación clínica en tuberculosis y micobacteriosis no tuberculosa. Hospital Pablo Tobón Uribe, enero 2004-diciembre 2011

\begin{tabular}{|lcccc} 
Presentación clínica & \multicolumn{2}{c}{ Tuberculosis } & \multicolumn{2}{c}{ MNT } \\
& n: $\mathbf{1 7 0}$ & $\mathbf{\%}$ & n: $\mathbf{1 7}$ & \multicolumn{1}{c}{$\%$} \\
Pulmonar & 85 & 50,00 & 4 & 23,5 \\
Extrapulmonar & 55 & 32,35 & 7 & 41,1 \\
Pleural & 18 & 10,58 & & \\
Ganglionar & 13 & 7,64 & 1 & 5,8 \\
Gastrointestinal & 11 & 6,47 & 2 & 11,7 \\
Hueso & 7 & 4,11 & 1 & 5,8 \\
Piel y tejidos blandos & 2 & 1,17 & 3 & 17,6 \\
Sistema nervioso central & 3 & 1,76 & & \\
Genito-urinario & 1 & 0,58 & & \\
Diseminada & 30 & 17,64 & 6 & 35,2 \\
\hline
\end{tabular}

Tabla 3. Especies de micobacterias aisladas en el Hospital Pablo Tobón Uribe, enero 2004-diciembre 2011 (n: 187)

$\begin{array}{lrc}\text { Especie de micobacteria } & \mathbf{n} & \% \\ \text { Complejo Mycobacterium tuberculosis } & 170 & 90,9 \\ \text { M. tuberculosis } & 168 & 89,83 \\ \text { M. bovis } & 2 & 1,07 \\ \text { Micobacterias no tuberculosas (MNT) } & 17 & 9,1 \% \\ & \mathbf{n} & \% \\ \text { Especies de MNT } & 17 & 100 \\ \text { Complejo Mycobacterium avium } & 6 & 35,29 \\ \text { Mycobacterium abscessus } & 3 & 17,65 \\ \text { Mycobacterium chelonae } & 2 & 11,76 \\ \text { Mycobacterium scrofulaceum } & 1 & 5,88 \\ \text { Mycobacterium kansasii } & 1 & 5,88 \\ \text { Mycobacterium malmoense } & 1 & 5,88 \\ \text { Mycobacterium immunogenum } & 1 & 5,88 \\ \text { Mycobacterium marinum } & 1 & 5,88 \\ \text { Mycobacterium fortuitum } & 1 & 5,88\end{array}$

HPTU: Hospital Pablo Tobón Uribe. MNT: micobacterias no tuberculosas.

Tabla 4. Perfil de susceptibilidad en $\boldsymbol{M}$. tuberculosis aislados en el Hospital Pablo Tobón Uribe, enero 2004-diciembre 2011 (n: 47)

\section{Perfil de susceptibilidad}

Frecuencia

Sensible a fármacos de primera línea

$55 \%$

Resistencia a estreptomicina

MDR

Resistencia a isoniazida

Resistencia a etambutol + rifampicina

Resistencia a estreptomicina + rifampicina

Resistencia a estreptomicina + isoniazida

XDR

$15 \%$

$11 \%$

$4 \%$

$4 \%$

$4 \%$

$4 \%$

$2 \%$

MDR: Multidrogoresistente (resistencia a isoniazida y rifampicina). XDR: Resistencia extendida (MDR + resistencia a aminoglucósidos y quinolonas).
El 58,8\% fueron hombres. Los factores de riesgo, las co-morbilidades y los medicamentos inmunosupresores usados por estos pacientes se presentan en la Tabla 1 . El compromiso pulmonar fue la forma de presentación clínica más frecuente (Tabla 2).

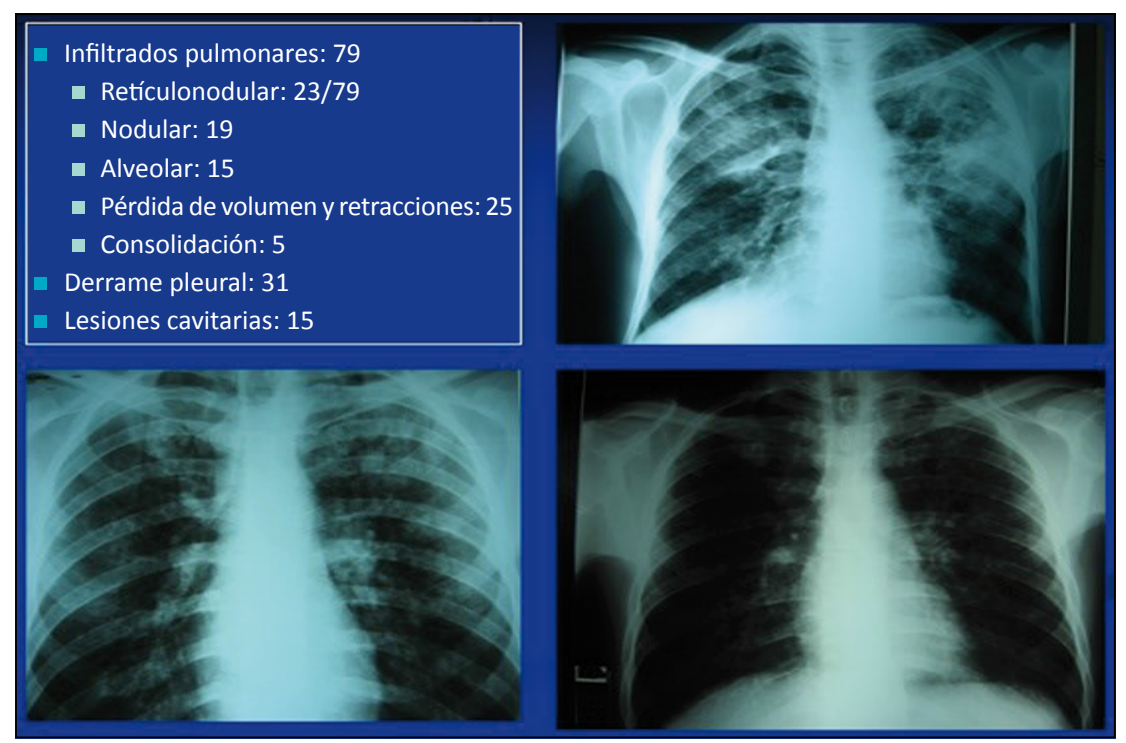

Figura 1. Características radiográficas. Radiografía del tórax: Las principales manifestaciones radiográficas fueron infiltrados pulmonares, efusión pleural y lesiones cavitarias. En el parénquima predominaros los infiltrado retículo nodulares y nodulares, seguidos de pérdida de volumen, retracciones e infiltrados de ocupación alveolar.

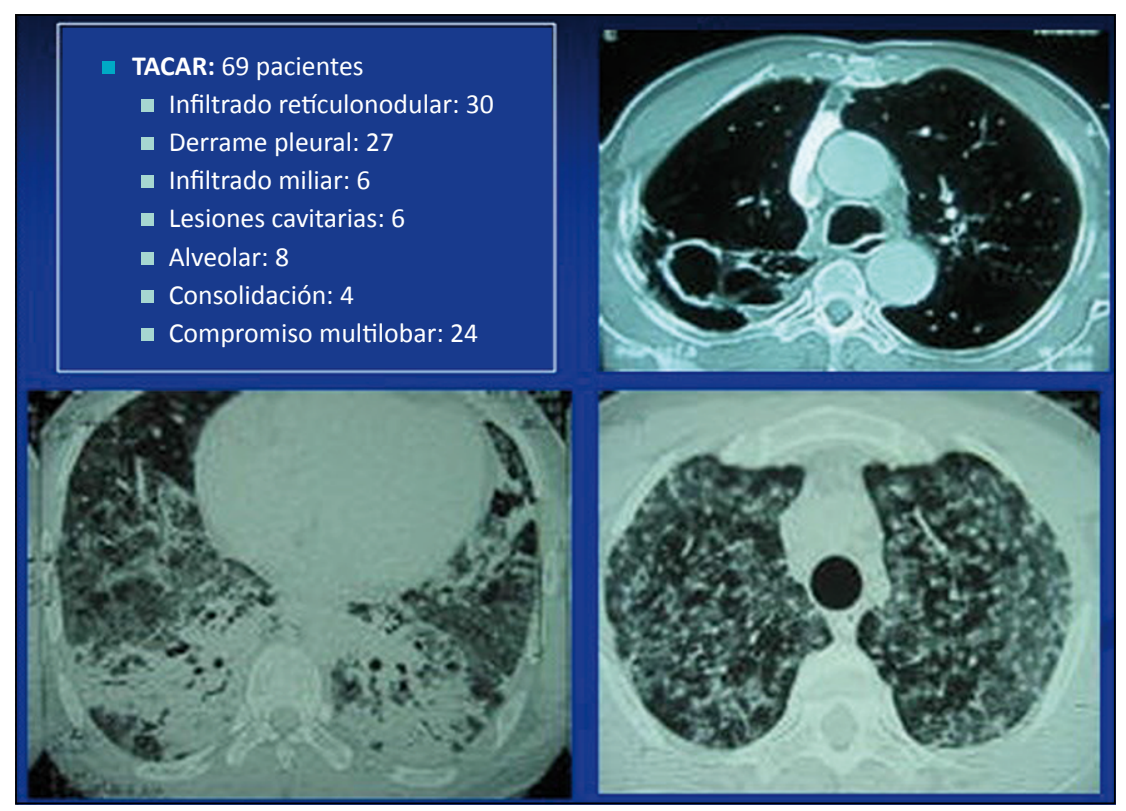

Figura 2. Características tomográficas. Tomografía del tórax: Los más importantes hallazgos fueron infiltrados retículo nodulares, efusión pleural, infiltrados nodulares de aspecto miliar, lesiones cavitarias, infiltrados alveolares y consolidación. Compromiso multilobar fue identificado en 35\%. 
La mediana de estancia hospitalaria fue de 20 días (rango 0-133); 23,5\% requirió atención en UCI con una mediana de estancia en ella de 7 días (rango 1-47) y la mortalidad fue de $23,5 \%$. Todas las muertes ocurrieron en pacientes que recibieron atención en UCI.

Siete de los 15 pacientes en los que se cuenta con esta información estaban co-infectados con VIH, con un promedio de recuento de LTCD4 de 25 céls $/ \mathrm{ml}$ (rango 1-50) y una carga viral promedio de 83.345 copias $/ \mathrm{ml}$ (rango 40-1.253.817). En los seis que tenían estos datos, los LTCD4 fueron menores de 50 en $85,7 \%$.

Las especies más frecuentes fueron complejo M. avium (CMA) 35,3\%, M. abscessus 17,7\% y M. chelonae en $11,8 \%$ (Tabla 3). La mortalidad fue de $26,7 \%$, (cuatro de los 15 de quienes se contó con la información). En las infecciones por MNT no se procesaron pruebas de susceptibilidad porque no estaban disponibles a la fecha del estudio.

De los 12 pacientes a quienes se les realizó $\mathrm{Rx}$ de tórax al ingreso, $8(66,7 \%)$ no evidenciaban alteraciones. Dos $(16,7 \%)$ presentaban infiltrados alveolares, uno $(8,3 \%)$ con infiltrados mixtos y otro $(8,3 \%)$ reticulares. Los dos pacientes en quienes se obtuvo una TACAR, presentaron infiltrado nodular difuso.

Se realizó TAC y/o RM de cráneo a tres pacientes y se identificaron lesiones en anillos en uno, leucoencefalopatía en otro e infartos en el tercero.

De 14 pacientes de los cuales se cuenta con la información, uno $(7,1 \%)$ presentó falla respiratoria, ninguno presentó hepatotoxicidad o falla renal medicamentosa ni falla orgánica múltiple.

\section{Discusión}

A pesar de que Colombia es un país endémico para $\mathrm{TBC}$, son pocos los reportes conocidos respecto a las características socio-demográficas, clínicas y microbiológicas de los pacientes hospitalizados con infección por micobacterias. En nuestra serie, los hombres fueron más afectados que las mujeres, tanto por $M$. tuberculosis como en MNT, hallazgo compatible con lo descrito en las series mundiales ${ }^{12,15}$. El abuso de sustancias psicoactivas es el factor de riesgo conductual más comúnmente reportado entre los pacientes con $\mathrm{TBC}^{7,16}$, contrario a nuestro estudio en el que se encontró una alta prevalencia de consumo de alcohol y tabaquismo, y en muy pocos casos uso de drogas inhaladas o intravenosas. Se ha reportado que el riesgo de TBC entre los pacientes con enfermedad renal crónica es 6,9 a 52,5 veces mayor que en individuos sin enfermedad renal $^{17}$; en nuestra serie se encontró una prevalencia de enfermedad renal crónica de 9,62\%.

De acuerdo a datos de la OMS, $15 \%$ de los pacientes con TBC pueden tener co-infección con $\mathrm{VIH}^{1,18}$ y la tasa de co-infección esperada en la población colombiana oscila entre 10 y $15 \%$, datos que son sobrepasados en mucho en nuestra serie, donde pudimos documentar que la co-infección fue de 27,3\%. El riesgo de desarrollar TBC activa en un paciente con infección por VIH que tenga TBC latente (PPD positiva) se incrementa 100 veces y el riesgo anual es de 7 al 10\%, comparado con el riesgo de desarrollarla en un paciente no infectado por $\mathrm{VIH}$, que es del $10 \%$ a lo largo de toda su vida. En 30 a 35\% de los casos de TBC en pacientes con VIH, ésta es considerada como TBC primaria ${ }^{1,9,18}$.

La normatividad colombiana exige que a todo paciente con infección por TBC se le realice prueba para detectar el VIH, situación de obligatorio cumplimiento en nuestra institución, previo consentimiento informado del paciente y que se ve reflejada en nuestra serie donde el porcentaje de co-infección encontrado fue mucho mayor $(27,3 \%)^{1,2-}$ ${ }^{4}$. La co-infección con VIH facilita la presentación de formas extra-pulmonares y diseminadas, como se pudo corroborar en nuestra serie y como se describe también en el estudio de Ong y cols., donde de 480 casos de TBC extra-pulmonar, $76 \%$ tuvieron cultivo positivo y por análisis multivariado encontraron como factores de riesgo para TBC extra-pulmonar el ser jóvenes, de sexo femenino y la co-infección por VIH. Los principales compromisos fueron: ganglionar (45\%), osteo-articular (16\%), pleural $(14 \%)$, genitourinario $(8 \%)$, peritoneal $(5 \%)$, meníngeo $(2 \%)$ y diseminada $(2 \%)^{19}$. En nuestra serie, las formas extra-pulmonares más frecuentes fueron la ganglionar $(18,2 \%)$, gastrointestinal $(6,8 \%)$, ósea $(4,5 \%)$, meníngea $(4,5 \%)$ y cutánea $(2,3 \%)$ y cuando comparamos la población co-infectada con VIH versus los no infectados, encontramos que las formas extra-pulmonares y diseminadas fueron más frecuentes en la población co-infectada $(42,6$ vs $13,5 \%$ p < 0,001) y la forma pulmonar la más frecuente en la población sin infección por VIH $(65,9$ vs $21 \%, \mathrm{p}<0,001)$.

A medida que la incidencia de TBC disminuye en áreas del mundo donde las condiciones socio-económicas mejoran, la frecuencia de aislamiento de MNT aumenta y su relevancia en enfermedades humanas se hace más evidente. En contraste con M. tuberculosis, cuyo reporte es obligatorio en el mundo, las infecciones por MNT no se reportan sistemáticamente, por lo que se carece de datos válidos acerca de su verdadera incidencia y prevalencia. Sin embargo, en los países de alta prevalencia de TBC se describen cada día más casos de infecciones por MNT y esto ha generado interés por la descripción de las mismas así como de optimizar los métodos de detección e implementación de pruebas de biología molecular ${ }^{12,13,20}$. En nuestra serie se encontró una prevalencia de enfermedad por MNT de 9,1\%. Las infecciones por MNT tienen la inmunosupresión como el principal factor de riesgo; en nuestra serie todos los pacientes cursaron con inmunosupresión de diversas causas, la principal de ellas fue la infección por VIH. 
En el último estudio de vigilancia de la resistencia a fármacos anti-tuberculosos realizado en Colombia se reportó un porcentaje de $\mathrm{MDR}$ de $2,38 \%{ }^{21}$; en nuestra serie encontramos que en los pacientes con TBC, sólo a 47 de $170(27,6 \%)$ se les realizó pruebas de susceptibilidad, $10,6 \%$ fueron catalogados como TBC-MDR y $2,12 \%$ como XDR. Llama la atención además la alta resistencia a alguno de los fármacos anti-TBC de primera línea en $36,17 \%$ de los casos y dentro de éstos, el alto porcentaje de resistencia primaria a estreptomicina $(15 \%)$, medicamento que estuvo incluido hasta hace varios años en el esquema de tratamiento de primera línea de la TBC en nuestro sistema de seguridad social. El perfil de resistencia documentado en nuestra serie y diferente de los reportes nacionales, consideramos que está influenciado por las características de nuestra población: pacientes hospitalizados y gravemente enfermos, donde la co-morbilidad y la inmunosupresión son importantes ${ }^{3,4,21}$.

La mortalidad general en nuestros pacientes fue de 9,09\%; sin embargo, cuando comparamos la mortalidad por TBC con la mortalidad por MNT, hubo una diferencia significativa para MNT, donde la mortalidad fue de $23,53 \%$, expresando posiblemente el grado de complejidad y co-morbilidad de los pacientes atendidos en nuestra institución. La alta mortalidad identificada en nuestra serie, tanto general, como la mortalidad intrahospitalaria, fue superior a la reportada en otros estudios de América Latina ${ }^{1,22}$, posiblemente también asociada a la alta prevalencia de co-infección con VIH, así como el grado de inmunosupresión de los pacientes con infección VIH con recuentos de LTCD4 bajos, que está asociado a formas más graves de enfermedad, a formas diseminadas y a mayor mortalidad en este grupo de pacientes ${ }^{23}$.

Nuestro estudio tiene fortalezas como es el de aportar datos epidemiológicos regionales y para las Américas de la relación existente entre las diferentes formas de presentación clínica y la co-morbilidad y como éstas impactan la mortalidad. De igual forma, el poder documentar que la infección por MNT no es infrecuente en nuestras poblaciones y que debemos tenerlas siempre presentes en el diagnóstico diferencial, especialmente en pacientes inmunosuprimidos.

Igualmente, la serie presenta debilidades, pues el carácter retrospectivo de los datos lleva a que se pierda información importante y adicionalmente nuestros datos no se puedan extrapolar a pacientes ambulatorios.

\section{Conclusión}

Las infecciones por micobacterias en pacientes hospitalizados, y especialmente en pacientes inmunsuprimidos, tienen un comportamiento diferente al de la población ambulatoria, con formas de presentación clínica más graves, con mayor incidencia de formas extra-pulmonares y diseminadas así como mayor mortalidad. La resistencia a fármacos en este grupo de pacientes es muy llamativa, con un alto porcentaje de resistencia a fármacos de primera línea, así como de MDR y XDR, que difieren de los estudios nacionales. Las infecciones por MNT están presentes en nuestro medio y debemos hacer todos los esfuerzos por diagnosticarlas

\section{Resumen}

Introducción: Tuberculosis (TBC) es aún una entidad de alta prevalencia y mortalidad en el mundo. La resistencia ascendente a fármacos es un problema de salud pública. Además se describen con mayor frecuencia infecciones por micobacterias no tuberculosas (MNT) en áreas de alta prevalencia de TBC. Objetivos: Determinar características epidemiológicas, clínicas y microbiológicas de las infecciones por micobacterias documentadas por cultivo. Materiales y Métodos: Estudio observacional, descriptivo, en pacientes hospitalizados. Resultados: De 187 pacientes, en $90,9 \%$ se identificó complejo $M$. tuberculosis y en 9,1\% MNT; 64\% fueron hombres. Edad promedio 40 años (rango 1-88 años). Las principales co-morbilidades fueron infección por VIH/SIDA $(23,5 \%)$, uso de corticoesteroides $(13,3 \%)$ y enfermedad renal crónica $(9,6 \%)$. Las formas clínicas fueron pulmonares $(56,6 \%)$, extra-pulmonares $(23,9 \%)$ y diseminadas $(19,2 \%)$. El compromiso extra-pulmonar más frecuente fue ganglionar $(7,4 \%)$ y gastrointestinal (7\%). En $M$. tuberculosis 10,6\% fueron multidrogoresistentes (MDR) y $2,12 \%$ con resistencia extendida (XDR). Mycobacterium avium y M. abscessus fueron las MNT más frecuentes. La mortalidad general fue 10\%. Conclusiones: Inmunosupresión es el principal factor de riesgo para enfermedad extrapulmonar y/o diseminada y la resistencia a fármacos en pacientes hospitalizados con TBC es llamativa, con mayor incidencia de MDR y XDR. Las infecciones por MNT no son infrecuentes en nuestro medio.

\section{Referencias bibiográficas}

1.- World Health Organization. Global tuberculosis report 2013.www.who.int/tb/publications/ bobal_report/en/

2.- Secretaría seccional de Salud y Protección Social de Antioquia. Boletín temático de salud 2012.www.dssa.gov.co

3.- Instituto Nacional de Salud. Vivamos sin tuberculosis. Plan estratégico Colombia Libre de tuberculosis 2010-2015. Tercera edición, Diciembre 2009.

4.- Rodríguez D A, Gil N A, Vera N R. Tuberculosis situation in Colombia 2007- 2008 with emphasis in pediatric cases and coinfection TB-VIH. Infect 2010; 14 (3): 195-205.

5.- Jacob J T, Mehta A, Leonard M K. Acute forms of tuberculosis in adults. Am J Med 2009; 122: 12-7.

6.- Ladefoged K, Rendal T, Skifte T, Andersson M, Søborg B, Koch A. Risk factors for tuberculosis 
in Greenland: case-control study. Int J Tuberc Lung Dis 2011; 15(1): 44-9.

7.- $\quad$ Lin H H, Ezzati M, Chang H Y, Murray M. Association between tobacco smoking and active tuberculosis in Taiwan: prospective cohort study. Am J Respir Crit Care Med 2009; 180: 475-80.

8.- Gómez-Reino J J, Carmona L, Angel Descalzo M, Biobadaser Group. Risk of tuberculosis in patients treated with tumor necrosis factor antagonists due to incomplete prevention of reactivation of latent infection. Arthritis Rheum. 2007; 57(5): 756-61

9.- Sester M, van Leth F, Bruchfeld J, Bumbacea D, Cirillo DM, et al for the TBNET. Risk assessment of tuberculosis in immunocompromised patients - A TBNET Study. Am J Respir Crit Care Med 2014 Oct 10. DOI: $10.1164 / \mathrm{rccm} .201405-0967 \mathrm{OC}$

10.- Wright A, Zigno Ml, Van Deun A, Falzon D, Ruesch Gerdes S, Feldman K, et al. Epidemiology of antituberculosis drug resistance 2002-07: an updated analysis of the Global Project on Anti-Tuberculosis Drug Resistance Surveillance. Lancet 2009; 373: 1861-73.
11.- Madariaga MG, Lalloo UG, Swindells S. Extensively drug-resistant tuberculosis. Am J Med 2008; 121: 835-44.

12.- Glassroth J. Pulmonary disease due to nontuberculous Mycobacteria. Chest 2008; 133 : 243-51.

13.- Van Ingen J. Diagnosis of nontuberculous mycobacterial infections. Semin Respir Crit Care Med 2013; 34(1): 103-9. doi: 10.1055/s0033-1333569. Epub 2013 Mar

14.- World Health Organization. Multidrug and extensively drug-resistant $\mathrm{TB}(\mathrm{M} / \mathrm{XDR}$ TB).2010 Gobal report on surveillance and response. http://whqlibdoc.who.int/ publications/2010/9789241599191_eng.pdf

15.- Burzynski J, Schluger NW. The epidemiology of tuberculosis in the United States. Semin Respir Crit Care Med 2008; 29 (5): 492-8.

16.- Oeltmann J E, Kammerer J S, Pevzner E S, Moonan P K. Tuberculosis and substance abuse in the United States, 1997-2006. Arch Intern Med 2009; 169 (2): 189-97.

17.- Hussein M M, Mooij J M, Roujouleh H. Tuberculosis and chronic renal disease. Semin Dial 2003; 16 (1): 38-44.

18.- World Health Organization. Global tuberculosis control 2009: epidemiology, strategy, financing. Geneva: WHO; 2009 [acceso febrero12 de 2011]. Disponible en: http://www.who.int/tb/publications/global_ report/2009/en/index.html.

19.- Ong A, Creasman J, Hopexell P C, González L C, Wong M, Jasmer R M. A molecular epidemiological assessment of extrapumonary tuberculosis in San Francisco. Clin Infect Dis 2004; 38 (1): 25-31.

20.- Wolinsky E. Nontuberculous mycobacteria and associated diseases. Am Rev Respir Dis 1979; 119 (1): 107-59.

21.- Garzón M C, Angée D Y, Llerena C, Orjuela D L, Victoria J E. Vigilancia de la resistencia de $M$. tuberculosis a los fármacos antituberculosos, Colombia 2004-2005. Biomédica 2008; 28: 319-26.

22.- Bernabe-Ortiz A. Factores asociados a supervivencia en pacientes con tuberculosis en Lima Perú. Rev Chilena Infectol 2008; 25(2): 104-7

23.- Marchie T T, Akhigbe O T. Comparing the level of CD4 lymphocytes, to pulmonary features of tuberculosis in HIV patients in a local hospital. Niger J Clin Pract 2010; 13(3): 2549-9. 